Illinois State University

ISU ReD: Research and eData

Theses and Dissertations

2-10-2021

\title{
The Effect Of A Text-Message Based Hydration Education Intervention On Hydration Knowledge And Behavior Of Division I Female Collegiate Soccer Players
}

Milaena Ferrarese

Illinois State University, meferra@ilstu.edu

Follow this and additional works at: https://ir.library.illinoisstate.edu/etd

Part of the Kinesiology Commons, and the Public Health Education and Promotion Commons

\section{Recommended Citation}

Ferrarese, Milaena, "The Effect Of A Text-Message Based Hydration Education Intervention On Hydration Knowledge And Behavior Of Division I Female Collegiate Soccer Players" (2021). Theses and Dissertations. 1365.

https://ir.library.illinoisstate.edu/etd/1365

This Thesis is brought to you for free and open access by ISU ReD: Research and eData. It has been accepted for inclusion in Theses and Dissertations by an authorized administrator of ISU ReD: Research and eData. For more information, please contact ISUReD@ilstu.edu. 


\section{THE EFFECT OF A TEXT-MESSAGE BASED HYDRATION EDUCATION \\ INTERVENTION ON HYDRATION KNOWLEDGE AND BEHAVIOR \\ OF DIVISION I FEMALE COLLEGIATE SOCCER PLAYERS}

\section{MILAENA FERRARESE}

\section{Pages}

There is increasing evidence of athletes entering their training sessions in an already dehydrated state. It is crucial for athletes to properly replenish fluid and electrolytes lost via sweat in order to optimize their training and recovery. The purpose of this study was to assess the effect of a text-message based hydration education intervention on the knowledge and hydration status of division I female collegiate soccer players.

A total of 14 participants completed baseline testing, which included hydration knowledge and behavior questionnaires, and pre- and post-practice weights to calculate \% weight change (\%WC). The participants also provided a baseline urine sample pre- and postpractice to assess urine specific gravity (USG). A total of 18 text messages were sent during the 6-week intervention phase, with 3 text messages sent per week. The text messages contained educational information on hydration topics. Post-questionnaires were administered after the intervention online, via Qualtrics.

The averages of pre-training USG $(M=1.02, S D=.01)$ and post-training USG $(M=1.02$, $S D=.01)$, did not change from before to after a practice session. There were no significant correlations between pre- training USG and pre- total hydration knowledge scores ( $\mathrm{r}=$ - 
$.274, \mathrm{p}>.05)$, post- training USG and pre- total knowledge scores ( $\mathrm{r}=.011, \mathrm{p}>.05)$, and \%WC and pre- total scores ( $\mathrm{r}=-.184, \mathrm{p}>.05)$. Of the pre-knowledge questionnaire questions there was a significant, moderate, positive relationship between USG and the true/false question asking, 'An athlete should consume 7-10 fl oz of water or sports drink a couple hours before exercise' $(\mathrm{r}=0.55, \mathrm{p}=.04)$. There was a numerical increase in the total scores when comparing post questionnaire total scores $(M=84.62, S D=6.05)$ to the pre-questionnaire total scores $(M=79.91, S D=9.55)$, but it did not reach statistical significance $(\mathrm{p}=0.14)$.

This study showed that text message-based forms of communication are a feasible intervention that could show positive improvement in both hydration status and hydration knowledge of athletes.

KEYWORDS: Hydration, Knowledge, Behavior, Athlete, Text intervention 


\title{
THE EFFECT OF A TEXT-MESSAGE BASED HYDRATION EDUCATION INTERVENTION ON HYDRATION KNOWLEDGE AND BEHAVIOR OF DIVISION I FEMALE COLLEGIATE SOCCER PLAYERS
}

\author{
MILAENA FERRARESE
}

A Thesis Submitted in Partial Fulfillment of the Requirements for the Degree of

MASTER OF SCIENCE

Department of Family and Consumer Sciences

ILLINOIS STATE UNIVERSITY

2021 
Copyright 2021 Milaena Ferrarese 


\title{
THE EFFECT OF A TEXT-MESSAGE BASED HYDRATION EDUCATION INTERVENTION ON HYDRATION KNOWLEDGE AND BEHAVIOR OF DIVISION I FEMALE COLLEGIATE SOCCER PLAYERS
}

\author{
MILAENA FERRARESE
}

COMMITTEE MEMBERS

Jennifer Barnes, Chair

Julie Schumacher

Scott Pierce 


\section{CONTENTS}

\section{Page}

TABLES

iii

FIGURES

iv

CHAPTER I: INTRODUCTION

Methodology

Sampling procedure

Instrumentation

Intervention

Statistical Analysis

Results

Demographics

Baseline Averaged of Pre-Questionnaire Total Scores, Urine

Specific Gravity, \& Percent Weight Change

Correlations Between All Knowledge Questionnaire Scores, Urine

Specific Gravity, \& Percent Weight Change

Correlations Between All Pre-Behavior Questionnaire Scores, Urine

Specific Gravity, \& Percent Weight Change 
Independent Samples Between Pre- \& Post-Knowledge, Behavior, and Overall Total Questionnaire Scores

Discussion

CHAPTER II: LITERATURE REVIEW

Hydration \& Performance

Knowledge of Hydration

Rehydration Strategies

Education Efficacy Through Text Message

REFERENCES

APPENDIX A: HYDRATION KNOWLEDGE QUESTIONNAIRE 


\section{TABLES}

Table

Page

1. Year in School of Participants

2. Correlations Between Averages of Pre-Intervention Knowledge

Questionnaire Total Scores, Pre- and Post-Training Urine Specific Gravity, and Pre- and Post-Training Percent Weight Change

3. Correlations Between Pre-knowledge Questionnaires \& Urine Specific Gravity

4. Correlations Between Pre-behavior Questionnaire \& Urine Specific Gravity 


\section{FIGURES}

Figure

Page

1. Percentage of Participants That Answered Correctly on the Pre \& Post-Knowledge questionnaire 


\section{CHAPTER I: INTRODUCTION}

Euhydration is defined as being in a state of optimal, total intracellular and extracellular body water content (Olzinski, Beaumont, Toledo, 2019). There is a detrimental impact on an athlete's performance if they are not properly hydrated both pre- and post-training. An individual experiencing $>2 \%$ loss of body mass due to water loss can be classified as dehydrated (Olzinski, Beaumont., Toledo, 2019). A state of dehydration is commonly seen following bouts of intense exercise or training, so it is crucial for the athlete to properly replenish fluid and electrolytes lost via sweat in order to optimize recovery. An ideal rehydration drink should include sodium which is an important electrolyte that resides in the extracellular fluid and functions to help maintain fluid balance (Evans, James, Shirreffs, 2017). If a large amount of sodium is lost during exercise and not adequately replenished, water will move by diffusion into cells causing them to swell, which can result in severe adverse effects (Evans, James, Shirreffs, 2017). Electrolytes are important in serving many biological functions with sodium being responsible for the excitability and cellular permeability of the muscle, and potassium functioning to aid in protein and carbohydrate synthesis (Orrù, Imperlini, Nigro, 2018). An issue commonly seen in athletes is known as exercise-associated hyponatremia (EAH). EAH is characterized by low blood sodium concentration $(<130 \mathrm{mmol} / \mathrm{L})$ that can develop during or immediately following intense exercise (Hew-Butler, Loi, Pani, \& Rosner, 2017). Some of the consequences associated with EAH are muscle cramping, dizziness, and impaired performance.

Past research suggests athletes lack knowledge surrounding adequate fluid and electrolyte replacement after training. In these studies it was found that athletes do not have a complete understanding of what they should be drinking, how much they should be drinking, or how often they should be drinking (Ayotte, David, \& Corcoran, (2018), Abbey EL, Wright CJ, \& 
Kirkpatrick, (2017), \& Magee, Gallagher, McCormack, (2017)). Coupled with the lack of knowledge it was found that most athletes will rely on thirst to prompt them to consume fluids. Although this is effective during periods of rest it is not as effective during training when fluid needs are increased. A study found that when humans drink ad libitum while participating in training that prompts high sweat rates, they markedly under consume fluid (Kenefick 2018). This has the potential to further exacerbate hypohydration given that athletes may begin their training sessions in a somewhat dehydrated state (Ayotte, David, \& Corcoran, 2018). A better understanding of what dehydration is can help athletes become more self-aware of hydrationrelated concerns. A better understanding may also help to improve hydration strategies and can potentially lead to a reduced risk of injury and illness (Judge, Kumley, Bellar, 2016).

The composition of the fluid consumed is crucial to recovery with the amount of fluid consumed being equally as important. When fluid intake matches sweat loss, it is vital that there is some type of simultaneous sodium replacement strategy in place as well. This practice is exercised in order to avoid the risk of developing reduced blood sodium or hyponatremia. Fluid replacement strategies will vary between person to person as there is no defined universal strategy to optimally replenish fluid and electrolytes. The reason for this is that people sweat at different rates and lose different amounts of sodium during training (Ayotte, David, \& Corcoran, 2018). It is important to figure out an individualized strategy that aligns with the type, duration, and intensity of exercise.

The purpose of this study was to assess the effect of a text-message based hydration education intervention on the knowledge and hydration status of female collegiate soccer players. The hypothesis was that the text-message intervention will improve the hydration knowledge of athletes, as well as their hydration status. 


\section{Methodology}

\section{Sampling Procedure}

The sample of this prospective study included 14 female participants from a division I collegiate soccer team, with ages ranging between 18-21 years old. All potential participants were informed that participation was voluntary. Whether or not they chose to participate had no impact on their standing with the university or athletics.

\section{Instrumentation}

The hydration knowledge questionnaire was modified from validated questions based on the position statements from the American College of Sports Medicine and The National Athletic Trainers Association (Nichols, Jonnalagadda, Rosenbloom, 2005). The hydration behavior questionnaire was created based on the theory of planned behavior model (Ajzen, 2019). The pre- and post-intervention knowledge questionnaire aimed to gauge the participants' knowledge of hydration through 16 true/false questions. The 10-question hydration behavior questionnaire addressed the participants' current hydration practices. The use of these questionnaires measured change in knowledge and behavior before and after the text-based education intervention. Preintervention questionnaires were taken as hardcopy, but post-intervention questionnaires were collected electronically via Qualtrics due to the COVID-19 pandemic. Weights were taken at baseline pre- and post-practice prior to the intervention phase. Weights were collected individually with only the participant and an investigator from the study present in the athletic weight room. The weights were then used to calculate \% WC for each participant. The athletes were also provided with a specimen cup which was used to collect a baseline urine sample preand post-practice. Urine samples were collected individually in the privacy of a stall, with only 
the participant present and investigator present in the locker room. The USG was immediately tested by the investigators using an Atago Pen Refractometer then disposed of immediately.

\section{Intervention}

During the 6-week intervention period, text messages were sent out through an online program called EZ Texting. Texts were sent three times per week (in the morning, before practice), containing educational information on specific hydration topics. One of the three textmessages each week asked for a short, one-answer response. No in-person meetings with study personnel occurred during this 6-week time frame. At the end of the intervention, period postintervention hydration knowledge and post-intervention hydration behavior questionnaires were administered. These results were compared to the baseline results. Due to the COVID-19 pandemic, data was not able to be collected for urine samples or weights to compare to the baseline measures.

\section{Statistical Analysis}

All data analyses were performed using statistical software IBM SPSS statistics. Descriptive statistics were generated for demographic data of each participant's age and year in school. An independent samples t-test was used to compare the independent variables (pre- and post-questionnaire questions and total scores) for each participant. A Pearson correlation was done to investigate the relationship between pre- and post-questionnaire questions, prequestionnaire questions with baseline USG and percent weight change, and between baseline

USG and total percent weight change individually. Statistical significance was set at $\mathrm{p}<.05$. 


\section{Results}

\section{Demographics}

The sample of this prospective cohort study included 14 female participants from a division I collegiate soccer team. One participant was a freshman, four were sophomores, five were juniors, and four were seniors. Their ages ranged between 18-21 years old $(M=19.80, S D=$ 1.12) (Table 1).

Baseline Averages Of Pre-Questionnaire Total Score, Urine Specific Gravity, \& Percent Weight Change

The averages of baseline pre-training $\operatorname{USG}(M=1.02, S D=.01)$ and baseline post-training USG $(M=1.02, S D=.01)$ did not change. This indicates that the hydration status of remained the same from before to after the training session. There were six correlations conducted between pre-intervention questionnaire total scores and pre-training USG $(r=-.27, p=.34) \backslash$, pre-total scores and post-training USG $(r=.01, p=.97)$, pre-intervention questionnaire total scores and $\%$ WC $(r=-.18, p=.53)$, pre-training USG and post-training USG $(r=.42, p=.14)$, pre-training USG and $\% \mathrm{WC}(r=-.06, p=.84)$, and post-training USG and \% WC $(r=-.13, p=.67)$. Among those none were statistically significant (Table 2). This indicates that there was no relationship between the pre-intervention objective measures of hydration status and the total scores of the pre-intervention knowledge questionnaires. 


\section{Correlations Between all Knowledge Questionnaire Scores, Urine Specific Gravity, \& Percent Weight Change}

Pearson correlations were run between pre- and post-training USG, pre- and post-training $\% \mathrm{WC}$, and each individual pre- knowledge questionnaire question. There was a significant, moderate, positive relationship between USG and KQ11 $(r=.55, p=.04)$ which was a T/F question asking, 'An athlete should consume 7-10 fl oz of water or sports drink a couple hours before exercise'. The average answer for that question was $(M=1.11, S D=.32)$ which means that most participants answered this question correctly. All other correlations were not statistically significant $(p>.05)$ (Table 3).

Correlations Between All Pre-Behavior Questionnaire Scores, Urine Specific Gravity, \& Percent Weight Change

Pearson correlations were run between pre-training USG, \% $\%$, and the individual preintervention behavior questionnaire questions. There was a significant, moderate, positive relationship between pre-training USG and BQ1 $(r=.53, p=.05)$, and pre-training USG and BQ7 $(r=.59, p=.03)$. Both BQ1 'drinking a large amount of water 3-4 hours before training would be good' and BQ7 'I intend to consume fluids before, during, and after training sessions' were answered on a 1-10 Likert scale $(1=$ good/agree, $10=$ bad/disagree $)$. This means that participants answered higher on the Likert scale that positively correlated with their USG, which indicated that they were more dehydrated and had poor hydration behaviors. All other correlations were not statistically significant $(p>.05)$ (Table 4$)$. 


\section{Independent Samples Between Pre- and Post-Knowledge, Behavior, and Overall Total Questionnaire Total Scores}

Independent samples t-tests were run between all individual pre- and post-intervention knowledge questionnaire questions, pre- and post-intervention behavior questionnaire questions, and total scores. There was a numerical increase in the total scores when comparing postquestionnaire total scores $(M=84.62, S D=6.05)$ to the pre-questionnaire total scores $(M=79.91$, $S D=9.55$ ) but it did not reach statistical significance, $\mathrm{p}=.14$. Question KQ12 was a T/F question that asked 'When exercise lasts for $>1$ hour, athletes should consume a sports drink over water', with the mean scores for post-test KQ12 $(M=1.31, S D=.48)$ being significantly lower than the mean scores for pre-test KQ12 $(M=1.71, S D=.47), \mathrm{p}=.04$. This indicates that most participants answered this incorrectly on the pre-intervention questionnaire compared to the post-intervention questionnaire. All other independent samples t-tests were not statistically significant.

\section{Discussion}

Proper hydration status is an important variable in determining how well an athlete may perform during training, as well as how quickly they recover. Inconsistent hydration practices occur in athletes due to the gap in knowledge surrounding what type of fluid to consume, how much fluid to consume, and when to consume fluid before, during, and after training. The hydration status and hydration behavior questionnaires showed a numerically positive change, which could be attributed to the intervention of electronic reminders about hydration. However, this numerical increase was not statistically significant.

Athletes often enter training sessions already dehydrated, only to further exacerbate their hydration status by not consuming adequate amounts of fluid during and after practice. In a study looking at volleyball players, a major finding was that a large number of participants entered into 
practice in a hypohydated state (32-64\% of participants), with a fluid consumption well below the National Athletic Trainers Association (NATA) recommendation (Cleary, Hetzler, Wasson, 2012). The participants in this study also had objective evidence of pre-practice hypohydration according to pre-intervention USG numbers $(M=1.02, S D=.01)$. The National Athletic Trainers Association states that a USG of less than or equal to 1.020 is acceptable for a well-hydrated individual (Osterberg, Horswill, \& Baker, 2009). A possible reason for this common trend of entering into practice hypohydrated may be due to the extreme knowledge gap surrounding hydration specifically "when to consume fluids and how much fluid to consume" (Trakman, Forsyth, Hoye, 2018).

In a study involving London marathon runners, the participants were asked to complete questionnaires that assessed their hydration strategies pre-, during, and post-marathon, and their sources of hydration information (Williams, Brown, Malliaras, 2012). The investigation found most participants reported a planned intake volume well above $3.5 \mathrm{~L}$ of water, which is the maximum intake before water intoxication. This number is relative to the weight of the person and other variables such as activity level. It is possible that these participants over reported their planned fluid intakes, which could confound the data. This relates to the current study because most of the participants from this study sample reported high fluid consumption before and during training, but the baseline USG recorded still showed most of the athletes to be in a dehydrated state.

There are specific hydration goals pre-, during, and post-training that are designed to optimize overall performance and recovery. The goal of $\sim 5-10 \mathrm{ml}$ fluid/kg body weight prior to training helps to ensure adequate hydration when entering a training session. (McDermott, B. P., Anderson, Armstrong, 2017). Fluid consumption during exercise is encouraged to avoid no more 
than a $2 \%$ decrease in body mass, which is when complications of dehydration may start to appear and negatively impact performance. Post-training fluid consumption is necessary to restore a state of euhydration and improve recovery, while reducing fatigue. Ideal consumption of $\sim 150 \%$ of fluid loss is recommended to fully restore hydration status (McDermott, Anderson, Armstrong, 2017). In this current study the researchers were unable to document postintervention USG and total \% WC due to the unexpected change in methods, caused by the COVID-19 pandemic. Athletes were no longer engaging in face-to-face training and researchers did not have access to participants for objective post-intervention data collection.

A study with 59 young elite athletes from various sports teams investigated the prevalence of hypohydration during a typical training day for an entire week. Hydration status was assessed using both USG and urine color. Results found that $89.8 \%$ of the participants were hypohydrated via USG, $96.6 \%$ were hypohydated via urine color chart, and only $23.7 \%$ of the athletes went in to practice adequately hydrated (Arnaoutis, Kavouras, Angelopoulou, 2015). Researchers are still trying to develop a concrete recommendation for how athletes can properly hydrate before, during, and after training to ensure adequate performance and recovery. A lot of sports drinks market themselves as "functional beverages" to help athletes restore electrolytes, carbohydrates, and other nutrients lost during exercise. These drinks are specifically made to contain essential electrolytes such as sodium, potassium, calcium, and magnesium which are depleted via sweat (Orrù, Imperlini, Nigro, 2018). As more education is presented to young athletes on the importance of replenishing fluid and electrolytes, the market for functional beverages continues to grow. When addressing the topic of fluid replacement strategies, it is important to emphasize that not only is the amount of fluid important, but the composition of the fluid is equally as important to consider. 
The literature has investigated various hydration education intervention programs that all differed in length and structure. There is currently no known length of intervention that has produced significant, long-term improvement in a participant's hydration knowledge and behavior. Text-message based interventions have become more popular with the recent advancements in technology. Studies utilizing this method of communication have shown promising results especially in the healthcare field (Abroms, Whittaker, Free, 2015; Schwebel, \& Larimer, 2018). There was a study done with 710 coronary heart disease patients, where cardiovascular risk factors were analyzed following a lifestyle-focused semi-personalized support program administered via text-message. The primary finding of this investigation was that the text-based intervention resulted in significant improvement of pertinent biochemical markers associated with coronary heart disease. The communication with participants through text-message was very efficient because the messages were sent directly to the individual at any time of the day. By receiving constant reminders, the likelihood of the recipient to follow through may be higher. There is a lack of studies in the literature that look at text-message based interventions in athletes. Most of the studies investigate older adults' adherence to physical activity. More research needs to be done in the athletic population on the use of a text-message based intervention and the overall outcome of the intervention on specific behaviors.

In this current study, the use of text-messaging to deliver information to the participants was an easy, cost effective method that was accessible to everyone. The information was sent early in the morning before practice with the intent that participants would remember to drink fluid before entering training. One day was designed to be an 'interactive' text-message, where the participants were asked to answer a simple "true/false" or "yes/no" question. This engagement allowed the participants to further think about their hydration habits. In this current 
study the consistent reminders may have impacted the results of the hydration knowledge questionnaires slightly with the numerical increase in total scores post-intervention, indicating that the intervention may have had a positive influence on the overall outcomes. However, the increase was not significant. It is unclear whether the intervention impacted the athlete's hydration status because the researchers were unable to measure the appropriate marker of hydration to compare to pre-intervention measures.

The strengths of the current study included the use of USG as a measure of hydration status at baseline, and the use of a text-message-based intervention program. USG is an objective measure that gives a more accurate depiction of the participants' hydration status, as it measures the concentration of the urine. Assessing urine color is a subjective tool that is often used, yet it is less accurate than measuring USG. This strengthens the validity of the overall study. Using text-message as a means of communication is a novel innovation and is continuing to rise in popularity. This allows participants to receive and engage in the intervention without having to contribute too much of their time. An advantage of using an interactive intervention is that it causes participants to focus on the topic and critically think about their hydration for a moment. When they actively participate it may increase the chances of improving their overall hydration knowledge and behaviors.

There were various limitations with the current study, however the biggest limitation was the unexpected occurrence of COVID-19. The pandemic began right in the middle of the intervention period, however, did not influence the intervention, as it was text-message based. During this time the athletes were still required to workout at home, all following the same workout schedule. The limitation arose when it was time to collect the post-intervention data. This warranted a switch in the methods to online, rather than hard copy questionnaires, and an 
inability to collect post-intervention \% WC or USG. Without the objective post-intervention data, it is impossible to compare the impact of the intervention on those specific variables of hydration status. Another weakness was the number of participants, with only 14 athletes completing the study. A low sample size can diminish the overall power of the study. Further, the participants were recruited from a team that has a reputation of being highly compliant and motivated which can be a confounding factor to assessing their behavior change.

This study showed the potential of using text-message based interventions to communicate with collegiate student athletes on improving hydration knowledge and behavior. Sending out a text-message any point during the day is a feasible way to reach participants in the study. As time goes on and technology evolves to be more advanced, there is a potential to move to a more complex way of communicating through technology. More research needs to be done to evaluate how hydration knowledge and status changes after administering a text-message based intervention. This study showed that this form of communication as an intervention could potentially result in a positive improvement in both hydration status and hydration knowledge of athletes. 
Table 1: Year in School of Participants

\begin{tabular}{l|c|c}
\hline \multicolumn{1}{c|}{$\begin{array}{c}\text { Year in } \\
\text { school }\end{array}$} & Percent & Frequency \\
\hline Freshman & $7.14 \%$ & 1 \\
Sophomore & $28.57 \%$ & 4 \\
Junior & $35.71 \%$ & 5 \\
Senior & $28.57 \%$ & 4 \\
\hline
\end{tabular}

Table 2. Correlations Between Averages of Pre-Intervention Knowledge Questionnaire Total Scores, Pre- and Post-Training Urine Specific Gravity, and Pre- and Post-Training Percent Weight Change

\begin{tabular}{|c|c|c|c|c|}
\hline Variable & Total score & Pre USG & Post USG & $\% \mathrm{WC}$ \\
\hline $\begin{array}{l}\text { Total } \\
\text { score }\end{array}$ & 1 & & & \\
\hline 2. Pre USG & -.27 & 1 & & \\
\hline 3. Post USG & .01 & .42 & 1 & \\
\hline 4. \% WC & -.18 & -.06 & -.13 & 1 \\
\hline
\end{tabular}

Table 3. Correlations Between Pre-Knowledge Questionnaire and Urine Specific Gravity

\begin{tabular}{|c|c|c|c|c|c|c|c|c|c|c|c|c|c|c|c|c|}
\hline Variables & 1 & 2 & 3 & 4 & 5 & 6 & 7 & 8 & 9 & 10 & 11 & 12 & 13 & 14 & 15 & 16 \\
\hline Pre USG & .13 & -.13 & .12 & .37 & N/A & N/A & N/A & N/A & .48 & .16 & $.55^{*}$ & .32 & N/A & N/A & N/A & N/A \\
\hline $\begin{array}{l}\text { Post } \\
\text { USG }\end{array}$ & .11 & .02 & .31 & -.31 & N/A & N/A & N/A & N/A & .18 & .05 & .16 & -.03 & N/A & N/A & N/A & N/A \\
\hline
\end{tabular}

Table 4. Correlations Between Pre-Behavior Questionnaire and Urine Specific Gravity

\begin{tabular}{l|l|l|l|l|l|l|l|l|l|l}
\hline Variables & 1 & 2 & 3 & 4 & 5 & 6 & 7 & 8 & 9 & 10 \\
\hline Pre USG & $\mathbf{. 5 3 4 *}$ & .177 & .457 & .213 & .368 & -.212 & $\mathbf{. 5 9 4 ^ { * }}$ & -.135 & .464 & -.170 \\
Post & .244 & .229 & .480 & .490 & .008 & -.129 & .140 & -.338 & .338 & -.152 \\
USG & & & & & & & & & & \\
\hline
\end{tabular}

*Indicates significance at the $\mathrm{p}<.05$ level 
Figure 1. Percentage of Participants That Answered Correctly On The Pre- and Post-Knowledge

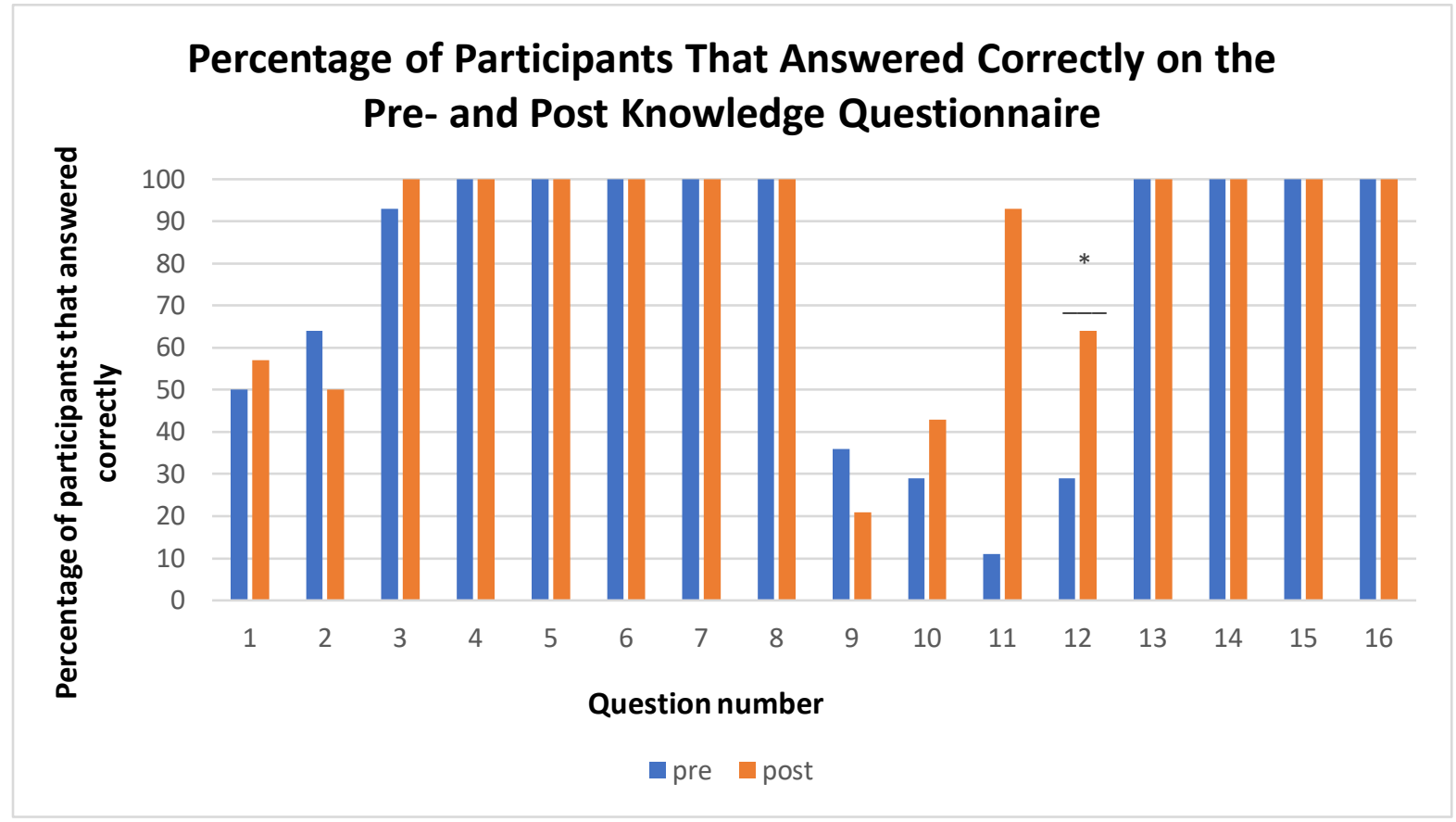

Questionnaire

*indicates significance at the $\mathrm{p}<.05$ level 


\section{CHAPTER II: LITERATURE REVIEW}

\section{Hypohydration and Performance}

Hypohydation can occur when there is a body water deficit, as a result of insufficient fluid consumption to match fluid loss. A factor contributing to hypohydration is thermoregulatory sweating, with the evaporation of sweat being the primary method of heat loss during exercise (Nuccio, Barnes, Carter, 2017). When beginning physical activity in an already dehydrated state, both anaerobic and aerobic components of performance are negatively impacted. These components include maximal oxygen uptake, anaerobic output, and physical work capacity (Yoshida, Takanishi, Nakai, et al., 2002). Hypohydration can impair performance in both team and individual sports, with factors that increase a person's risk of becoming dehydrated including hot/humid environments, exercise intensity, and inadequate fluid replacement strategies (Nuccio, Barnes, Carter, 2017). A study was done in 11 male soccer players that assessed whether or not moderate water loss ( $2 \%$ of body mass) had a significant impairment on sports performance and other fitness-related variables (Edwards, Mann, MarfellJones., 2007). The experimental phase was comprised of three different sessions: 1) a 45-minute pre-match period of cycling, 2) completion of a 45-minute soccer match, and 3) post-match performance of sport specific and mental concentration test. The procedure was carried out on three different occasions with the participants being in three different conditions (fluid intake (FL), no fluid (NF) and mouth rinse (MR)), all of which were randomized. The researchers analyzed core temperature, heart rates, urine osmolality, body mass, sweat rate, and heat storage. From the results it was concluded that the performance of a sport-specific fitness test was significantly impaired in the groups that did not consume any fluids. It also revealed that the 
rating of perceived exertion and thirst indication was most challenging in the non-fluid consumption groups (Edwards, Mann, Marfell-Jones., 2007).

Another study looked at 10 well-trained, male endurance cyclists that participated in an outdoor climbing trial in the heat, while in a state of mild dehydration. The objective was to determine if the hydration state of the cyclists had an influence on their performance during the trial (Bardis, Kavouras, Arnaoutis, 2013). The procedure was broken up into two different sessions with all participants performing in either a euhydrated state or a dehydrated state of $\sim 1 \%$ of body mass. The cycling exercise was performed on an ergometer with or without fluid intake, to achieve the desired pre-exercise dehydration state. The measurable outcomes of interest were performance time, core temperature, sweat rate/sensitivity, and rate of perceived exertion (Bardis, Kavouras, Arnaoutis, 2013). The results of the study revealed that performance time was faster in the euhydration group compared the dehydrated participants. Hypohydation resulted in a higher core temperature, lower sweat rate/sensitivity, and high perceived exertion compared to the euhydrated group (Bardis, Kavouras, Arnaoutis,2013).

A meta-analysis was done to quantify the effects of hypohydation on muscle performance variables, to determine how performance can be impaired by different hydration levels. After identifying several articles using database searches and cross-referencing, a total of 28 articles met the inclusion criteria for the study. The consensus was that overall muscle endurance was decreased by $8 \%$ in a state of hypohydation, with no significant difference between the upper and lower body (Savoie, Kenefick, Ely, et al., 2015). Muscle strength overall decreased by 5.5\% with a greater decrease in the lower muscle strength compared to upper body strength. Anaerobic power was significantly impaired by hypohydration, but the anaerobic capacity was not (Savoie, Kenefick, Ely, et al., 2015). 


\section{Knowledge of Hydration}

The literature suggests there is a lack of knowledge surrounding hydration status in the athletic environment (Trakman, Forsyth, Hoye, 2018). Knowledge questionnaires

are a valuable assessment tool when trying to determine where the gap in knowledge is, as it gives a general baseline as to how much an individual truly knows about a specific topic.

A study was conducted involving marathon runners participating in the London marathon. The objective of the study was to determine the hydration strategies of marathon runners, their sources of information and knowledge of fluid intake, as well as their understanding of exercise associated hyponatremia (EAH). There was a total of 217 runners who completed a questionnaire that consisted of questions pertaining to drinking strategies, sources of information about fluid intake, and knowledge of appropriate fluid intake (Williams, Brown, Malliaras, 2012). Results from the questionnaire showed that 199 of the 217 participants said they had a pre-race hydration plan for the morning of the marathon, which showcased their preparedness. Regarding fluid intake during the marathon, 208 out of 217 stated that they had a plan for fluid consumption during the race, with about half of them planning to drink from all 24 water stations. About $90 \%$ of the participants also established that they had a fluid intake strategy set in place for post-marathon recovery. Most of the runners gained their information on fluid intake from magazines, other running friends, running coaches, and the internet. Although most participants claimed to have a fluid intake strategy before, during, and after the race, many of the runners outlined an intake volume that was above $3.5 \mathrm{~L}$ of fluid which is the maximum amount of fluid intake before water intoxication. There is a chance that the participants over reported the amounts that they were drinking, which could alter the data and results of the study. This fluid amount may be dangerous and potentially put them at higher risk for developing a common 
phenomenon called exercise-associated hyponatremia (EAH). EAH happens when serum sodium levels fall below normal ranges during periods of intense physical activity. This can be further exacerbated with excessive fluid consumption without the replenishment of electrolytes and can cause adverse symptoms. The literature suggests that there is a lack of information regarding adequate hydration amounts to avoid the risk of EAH.

Another study looked at the effect of hydration education on college athlete's hydration status and hydration knowledge, attitudes, and practices (Sobana, \& Nirmala-Many, (2014). There was a total of 120 male and female athlete participants, with 60 serving as a control group and the other 60 as the intervention group. Non-intervention baseline anthropometric measures were taken a week before the intervention, and fluid intake was recorded for three days. A questionnaire was administered to assess hydration knowledge, attitude, and practices among the athletes. This was done at baseline and post-intervention to compare results. Hydration status was assessed using two distinct methods, urine color and percent change in body mass. The hydration education intervention covered topics including importance of fluid intake, dehydration symptoms, measure of hydration status, electrolytes, and sports drinks. The intervention phase was seven days for one hour each day, and provided information in the form of PowerPoint presentations, demonstrations, and exhibitions (Sobana, \& Nirmala-Many, (2014). The results report that the education program significantly improved the knowledge, attitude, and practices of the intervention group. Hydration status was tested again post-intervention and showed a significant improvement in hydration from baseline measures. The overall conclusion from this study was that hydration status had improved from baseline after receiving one week of continual hydration education. This highlighted the importance of 
hydration education and how it can teach athletes to make simple improvements in their hydration practices that benefit their overall performance and recovery.

There is no known length of hydration education that will produce significant long-term improvement in hydration knowledge and practice among athletes, and the literature has analyzed various types of education programs ranging in length. One study aimed to assess hydration status and behaviors among female athletes before and after a one-time educational intervention and prescribed hydration intervention in a warm, humid, and tropical environment (Cleary, Hetzler, Wasson, 2012). The study was done using thirty-six elite volleyball players and consisted of four observational periods: 1) control period (CP), 2) educational intervention (EI), 3) prescribed hydration intervention (PHI), 4) and observational follow-up period (OF-U). Each of the periods consisted of threepractices (Monday, Wednesday, Friday), which data was collected both before and after practice. The $\mathrm{CP}$ helped to establish the ad libitum hydration consumption among all participants, to give researchers a general idea of baseline practices.

During this period, researchers also conducted sweat rate calculations for each participant to be used during the PHI period. The EI required athletes to watch educational slideshows that provided them with hydration recommendations derived from the National Athletic Trainers' Association (NATA), they were given additional handouts to help reiterate the information that was already provided to them. The PHI phase took the sweat rate of each participant and designated a specific volume of water to consume during their practice, and if desired they were encouraged to consume more than the given amount. The OF-U period was void of any experimental treatments as it was used to determine how the previous experimental periods affected the hydration behaviors of participants. During this phase body mass, urine specific gravity (USG), osmolality, and urine color were all recorded. 
A major finding from this study was that many participants came to practice in an already hypohydated state (32-64\%) which was a major concern, considering the volume of fluid consumption was well below the NATA recommendation. Implementing a one-time educational intervention was not an appropriate duration to see changes in behavior status of athletes, although during the EI period there was a decrease in the number of participants reporting to practice in a hypohydated state. Implementation of a longer educational program coupled with a prescribed drinking volume during practice might show a more significant effect on an athlete's hydration knowledge and behavior.

\section{Rehydration Strategies}

Rehydration is important in maintaining hydration status and optimizing recovery (McDermott,B, Casa, \& Yeargin, 2009). It is critical that appropriate rehydration strategies are put into place to ensure an adequate replacement of fluid that an athlete loses during a training session. Commonly, the consumption of fluid does not match up to the amount an athlete sweats (Bergeron, Waller, \& Marinik, 2006). A beneficial tool that is used to assess how much fluid is lost during physical activity is the sweat rate calculation. An individual's sweat rate can vary day-to-day as well as person-to-person, with several variables contributing to the amount of sweat produced. To properly calculate the sweat rate, researchers need to know how much weight was lost during the training session, the amount of fluid consumed during the training session, and how long the training session lasted. Calculating the sweat rate can give a good idea of how much fluid is actually lost during a single training session and that can help facilitate a fluid replacement strategy that optimizes rehydration (McDermott, Anderson, Armstrong, 2017). Sweat rate is highly variable and the recommendations for fluid replacements are very individualized. 
The goal of hydration prior to exercise is to maximize safety and performance, which can be achieved at $\sim 5-10 \mathrm{ml} / \mathrm{kg}$ of fluid before training, depending on current hydration status (McDermott, Anderson, Armstrong, 2017). During exercise the goal is to consume enough fluid to avoid a $2 \%$ decrease in body mass, which is the level at which an individual can begin to experience complications of dehydration. It is also important to consider the consumption of sodium-containing beverages to maintain fluid balance. According to the American College of Sports Medicine (ACSM) most athletes will seldom consume adequate amounts of fluid to offset the amount lost through excessive sweat. They recommend a consumption of $\sim 6-12 \mathrm{oz}$ of fluid every 15-20 minutes to avoid the risk of becoming severely dehydrated (Convertino, Armstrong, Lawrence, Coyle, Edward ). If training lasts longer than one hour in duration, it would be beneficial to consume a carbohydrate and sodium-containing beverage (ideally a sports drink). The intended goal of post-exercise hydration is to restore euhydration to improve recovery and reduce fatigue. Up to $\sim 150 \%$ of estimated fluid loss is recommended to be consumed to adequately restore hydration status (McDermott, Anderson, Armstrong, 2017).

Another important consideration is the type of fluid that is being consumed. Rehydration drinks should include carbohydrates and electrolytes if exercise is scheduled to last longer than one hour which can help to maintain blood glucose, carbohydrate oxidation, and electrolyte balance (McDermott, Anderson, Armstrong, 2017). The recommended 6-8\% carbohydrateelectrolyte concentration ration of a beverage is beneficial to achieve the adequate restoration of muscle glycogen and electrolyte balance.

A study was conducted in 14 healthy male and female tennis players that were highly skilled, and training daily at an academy in central Florida. The overall protocol consisted of two test training days which entailed 120 minutes of intermittent sport-specific practice drills that 
caused an excessive amount of sweat (Bergeron, Waller, \& Marinik, 2006). During the sessions half of the group was asked to consume chilled unflavored water, and the other half consumed a chilled 6\% CHO-electrolyte drink. The crossover design called for the groups to switch drinks during the second testing. Both pre- and post-training hydration status was collected, and body weight was recorded before and after for analysis. The findings concluded that the tennis players came into each of the training sessions significantly dehydrated. The percent change in body weight helped the researchers conclude that most of these players did not match their sweat loss with the amount of fluid consumed during training. The most notable difference between the consumption of plain water compared to the $6 \% \mathrm{CHO}$-electrolyte drink was that the core temperature was lower in the participants drinking the sports drink (Bergeron, Waller, \& Marinik, 2006). This is relevant because the higher one's core temperature, the further one is from homeostasis and the more likely dehydration can occur.

A study done in 13 physically active males to observe the effect of a sodium-containing beverage on preventing hyponatremia, and muscle cramping. Exercise trials were performed in 3 phases, with each phase separated by a week. The first phase included 30 minute intervals between cycling and walking, the second phase included eight sets of standing calf raises, and the third phase included inclined walking on the treadmill for 45 minutes (Anastasiou, Kavouras, Arnaoutis, 2009). The participants were given four different test drinks during each of the exercise trials 1) a low-sodium $\mathrm{CHO}$ beverage, 2) a high-sodium $\mathrm{CHO}$ beverage, 3) artificially sweetened, flavored water, and 4) mineral water. Biochemical measures were taken to assess serum sodium, plasma osmolality/volume changes, and muscle cramping. Results showed that during trials when sodium-containing drinks were consumed, there was a slight increase in plasma osmolality and volume compared to the water beverages. Serum sodium concentrations 
were increased with the sodium-containing beverages and decreased with the water beverages.

Of the 13 participants, only four experienced muscle cramping which was reported during the trial where mineral water or artificially sweetened water was consumed (Anastasiou, Kavouras, Arnaoutis, 2009). Researchers found that sodium-containing beverages provided to athletes following an exercise-induced dehydration period had a significant impact on the restoration of body water loss, most specifically plasma volume. Another major takeaway of this study was that in environmental conditions, where fluid intake matches body mass loss, even the slightest amount of sodium in a beverage is adequate to attenuate the reduction in sodium concentrations and decrease the risk of hyponatremia (Anastasiou, Kavouras, Arnaoutis, 2009).

\section{Education Efficacy Through Text-Message}

As technology continues to advance, the use of mobile text-messaging has become a more popular method of delivering information to large scale populations. One can relay educational information, reminders, and facilitate behavior change all with one click of a button, making it relatively easy and efficient. Since technology is a huge part of society, there are benefits to using text-messaging such as the relatively low cost, user friendliness, and the ability to be sent directly to the individual at any time of the day (Schwebel, \& Larimer, 2018). Communication through text-messaging may also help to support specific individuals and remind them to engage in certain behaviors that will benefit their overall health (such as taking critical medications, attending their healthcare appointments, motivating them to take care of themselves, etc.). Along with the many benefits comes significant consequences if appropriate precautionary measures are not taken. The literature looks at text-message use within the healthcare system and frequently cites patient confidentiality as being a risk factor (Schwebel, \& Larimer, 2018). Unless the sender applied an encrypted messaging feature, there is a high 
possibility that the text-message being sent may be read by someone other than the intended receiver. Whether it be willingly or unwillingly, this is a risk that almost all individuals take daily. More research needs to be done on the appropriate timing, frequency, and dosage of textmessages that will be most effective in facilitating a specific behavior change. It is likely that these variables will vary amongst individuals, while taking into consideration the impact on the individual by the perceived importance of the message (Schwebel, \& Larimer, 2018).

There has been extensive research regarding text-messaging programs related to health behaviors in a variety of contexts including HIV prevention, medication adherence, pregnancy education, substance use/smoking cessation, weight loss, diabetes management, and depression (Abroms, Whittaker, Free, 2015). The use of text-messaging is seen as a useful tool to deliver information to patients in short time bursts throughout the day, and universal to most people across all income groups that have access to a mobile phone. More recently this communication platform has become acceptable in achieving public health goals in both large governmental health systems, and small-scale independent ones (Abroms, Whittaker, Free, 2015).

A parallel-group, single-blind, randomized clinical trial was conducted with 710 patients with proven coronary heart disease between September 2011 and November 2013. The aim of the study was to analyze the effect of a lifestyle-focused semi-personalized support program delivered via text-message on cardiovascular risk factors (Chow, Redfern, Hillis, 2015). The intervention group ( $\mathrm{N}=352)$ received a total of four text messages per week for a duration of six months, in addition to their usual healthcare routine. The text-messages were selected from a premade list of messages that were constructed on participant baseline characteristics, and served to provide patients with advice, motivational reminders, and support to change lifestyle behaviors. The participants in the control group $(\mathrm{N}=358)$ received their usual healthcare without 
the additional text-message information. Biochemical markers (i.e., fasting lipid levels, blood pressure, heart rate, BMI, and waist circumference) were tested for all participants at baseline and at the six months follow up period. The intervention participants also received a separate questionnaire that assessed the acceptability and perceived utility of the intervention. The primary outcome of the study was the decreased level of plasma LDL-C at 6 months, with all other biochemical markers being secondary outcomes. Results showed that LDL-C levels, systolic blood pressure, and BMI at the 6 months follow-up were significantly lower in the intervention group compared to the control group. Among the intervention group the large majority reported the text-message support program to be useful (91\%), easy to understand (97\%), and motivating in respect to diet (81\%) and physical activity change (73\%) (Chow, Redfern, Hillis, G, 2015). The overall conclusion of the study was that automated text- messages supporting lifestyle change compared to usual standard of care, led to a significant improvement in pertinent biochemical markers (i.e., LDL-C level, systolic blood pressure, and BMI) associated with coronary heart disease.

Another study that was done in the UK was a single-blind, randomized trial which aimed to assess the effect of an automated smoking cessation program, delivered via text-message, on the continuous abstinence of smoking at the six-month marker. There was a total of 5,800 participants who were randomized into the text intervention group $(\mathrm{N}=2,915)$ and the control group $(\mathrm{N}=2,885)$. The individuals in the intervention group received a total of five text-messages for the first five weeks and then three text-messages for the next 26 weeks. The information obtained in the text-messages were motivational and behavior change techniques, as well as promoting the use of QUIT smoking cessation telephone helplines and nicotine replacement 
therapy (Free, Knight, Robertson, 2011). The control group participants received fortnightly, simple, and short text-messages that were related to the importance of trial participation.

The primary outcome of this study was the self-reported continuous smoking abstinence that was confirmed at the six-month mark. Abstinence was defined as no more than five cigarettes smoked in the past week at the four weeks follow-up, and no more than five cigarettes smoked since the start of the abstinence period at the six months mark. The secondary outcomes of this study encompassed the prevalence of abstinence at both the 4-week follow-up and sixmonth mark. The researchers used postal salivary-cotinine testing to verify the self-reported abstinence of each participant at six months, with a cut off of $7 \mathrm{ng} / \mathrm{mL}$ (Free, Knight, Robertson, 2011).

The results of the study showed that the biochemically verified continuous abstinence at six months was significantly increased in the intervention group and doubled in self-reported smoking cessation at four weeks (pooled relative risk $[\mathrm{RR}] 2 \cdot 18,95 \%$ CI $1 \cdot 80-2 \cdot 65$ ). However, it is important to note that other smoking cessation support services were used by $50 \%$ of the intervention group. The consensus of the study was that the smoking cessation support via textmessaging doubled the quit rate at the six-month mark across all socioeconomic groups in younger and older smokers (Free, Knight, Robertson, 2011).

Text-message delivery is very effective in promoting behavior change, but the structure of the messaging system is crucial to the effectiveness of the program. The advantage of using text-message as a primary communication modality is that the information can be delivered quickly and in-the-moment of the decision making. An article was published to provide guidance on the steps it takes to develop a successful text-message intervention program (Abroms, Whittaker, Free, 2015). According to the research, an individual should set up their 
text-message system as if they were developing any typical health communication material. However, before attempting to develop a program, it is recommended to research the key behavior change mechanisms for the health behavior and population of interest (Abroms, Whittaker, Free, 2015). Once you have established a healthy behavior and target population, it is important to consider if a text-message program is even appropriate for that audience and if they have access to a mobile phone.

Once the bases are all covered, then it is time to consider the Communication Objectives and Behavioral Techniques, which will likely be based on theory (i.e., theory of planned behavior) and insights that you have developed through research. The Communication Objectives and Behavioral Techniques should be supported by certain components of the textmessage modality, as it is important to carefully plan out your message content around the construct of each theory that has been chosen. This is important groundwork that will help to set up a strong framework for the program, as it will be the "bulk" of the program and a large determinant of how successful the outcome will be. A few things must be considered during this phase of the process such as frequency of messages, timing of messages, and keywords that participants can use if they need additional assistance (Abroms, Whittaker, Free, 2015).

Message frequency should not overwhelm or burden the participants. It should be direct and to the point so that the researchers communicate only the key information. There is no recommended amount of text-messages that should be sent per day as it is going to be dependent upon the health behavior that is being investigated. The average frequency is around three textmessages per week, typically with one message per day. In the case of participants who have a high phone usage, the text-message frequency may be higher to ensure that they stand out among other text-messages in the users' inbox. Trying to delineate a perfect timing of text-messages 
should be based on the content of the message, the daily routine of the participant, and the nature of the behavior change (Abroms, Whittaker, Free, 2015). The goal is to send out the most pertinent messages with quality information, at a relatively appropriate time.

Another thing to consider is allowing participants to engage in the program by sending short responses back. That way they feel a more personal connection to the program and are more likely to facilitate their own behavior change. Responses should not be complex. Rather they should be simple, user friendly and easily interpreted so that the interaction remains positive. Lastly, there should always be some form of privacy protection so that the participants are not exposed to any risk. Text-messaging is not the most secure form of technology so there is some risk associated with the delivery of personal healthcare information that will originate from a Health Insurance Portability and Accountability Act (HIPAA) (Abroms, Whittaker, Free, 2015). It is crucial for the researcher to make these privacy risks clearly detailed in the terms and conditions provided to the participants before the start of any study.

The final steps in creating a successful text-message intervention program are to determine a message library of content to be sent out to the participants and pretest the finished product. Some general recommendations are to keep each message to 160 characters (including spaces) or less to be delivered as a single text-message to a mobile phone. If the message needs to be longer to accommodate additional important information, splitting the information into two separate messages is appropriate. The style and tone of the message should be professional and void of any slang or abbreviations, as participants generally find this to be unprofessional coming from a credible health source. It is also important to determine the literacy of the target audience. Some populations face a language barrier, so it might be beneficial to incorporate a translated version of the program to meet the demands of the participants trying to be reached. 
Lastly, the most important step is the pretesting phase to gain feedback on the specific components of the program. Using this constructive criticism, the researchers can make revisions as needed to improve the overall efficiency and success of the program. This is not just a onetime thing, generally it will take multiple rounds of feedback and revisions to create an adequate program. There are various ways to receive feedback through interviews, focus groups, and launching pilot testing of the program. The program should be constantly revised, improved, and retested. 


\section{REFERENCES}

Abbey EL, Wright CJ, Kirkpatrick CM. Nutrition practices and knowledge among NCAA Division III football players. J Int Soc Sports Nutr. 2017 May 19;14:13. doi:

10.1186/s12970-017-0170-2. PMID: 28529463; PMCID: PMC5437483

Abroms, L. C., Whittaker, R., Free, C., Mendel Van Alstyne, J., \& Schindler-Ruwisch, J. M. (2015). Developing and Pretesting a Text Messaging Program for Health Behavior Change: Recommended Steps. JMIR mHealth and uHealth, 3(4), e107. doi: $10.2196 /$ mhealth.4917

Ajzen, I. (2019). CONSTRUCTING A THEORY OF PLANNED BEHAVIOR QUESTIONNAIRE [Scholarly project]. Retrieved November 14, 2020, from https://people.umass.edu/aizen/pdf/tpb.measurement.pdf

Anastasiou, C. A., Kavouras, S. A., Arnaoutis, G., Gioxari, A., Kollia, M., Botoula, E., \& Sidossis, L. S. (2009). Sodium replacement and plasma sodium drop during exercise in the heat when fluid intake matches fluid loss. Journal of Athletic Training, 44(2), 117123. https://doi.org/10.4085/1062-6050-44.2.117

Arnaoutis, G., Kavouras, S. A., Angelopoulou, A., Skoulariki, C., Bismpikou, S., Mourtakos, S., \& Sidossis, L. S. (2015). Fluid Balance During Training in Elite Young Athletes of Different Sports. Journal of Strength and Conditioning Research, 29(12), 3447-3452. https://doi.org/10.1519/JSC.0000000000000400

Ayotte, D., Jr, \& Corcoran, M. P. (2018). Individualized hydration plans improve performance outcomes for collegiate athletes engaging in in-season training. Journal of the International Society of Sports Nutrition, 15(1), 27. https://doi.org/10.1186/s12970-0180230-2 
Bardis, C. N., Kavouras, S. A., Arnaoutis, G., Panagiotakos, D. B., \& Sidossis, L. S. (2013). Mild dehydration and cycling performance during 5-kilometer hill climbing. Journal of athletic training, 48(6), 741-747. https://doi.org/10.4085/1062-6050-48.5.01

Bergeron, M. F., Waller, J. L., \& Marinik, E. L. (2006). Voluntary fluid intake and core temperature responses in adolescent tennis players: sports beverage versus water. British Journal of Sports Medicine, 40(5), 406-410. https://doi.org/10.1136/bjsm.2005.023333

Chow, C. K., Redfern, J., Hillis, G. S., Thakkar, J., Santo, K., Hackett, M. L., Jan, S., Graves, N., de Keizer, L., Barry, T., Bompoint, S., Stepien, S., Whittaker, R., Rodgers, A., \& Thiagalingam, A. (2015). Effect of Lifestyle-Focused Text Messaging on Risk Factor Modification in Patients With Coronary Heart Disease: A Randomized Clinical Trial. JAMA, 314(12), 1255-1263. https://doi.org/10.1001/jama.2015.10945

Cleary, M. A., Hetzler, R. K., Wasson, D., Wages, J. J., Stickley, C., \& Kimura, I. F. (2012). Hydration behaviors before and after an educational and prescribed hydration intervention in adolescent athletes. Journal of Athletic Training, 47(3), 273-281. https://doi.org/10.4085/1062-6050-47.3.05

Convertino, Victor A. Ph.D., FACSM, (Chair); Armstrong, Lawrence E. Ph.D., FACSM; Coyle, Edward F. Ph.D., FACSM; Mack, Gary W. Ph.D.; Sawka, Michael N. Ph.D., FACSM; Senay, Leo C. Jr. Ph.D., FACSM; Sherman, W. Michael Ph.D., FACSM ACSM Position Stand: Exercise and Fluid Replacement, Medicine \& Science in Sports \& Exercise: October 1996 - Volume 28 - Issue 10 - p i-ix 
Free, C., Knight, R., Robertson, S., Whittaker, R., Edwards, P., Zhou, W., Rodgers, A., Cairns, J., Kenward, M. G., \& Roberts, I. (2011). Smoking cessation support delivered via mobile phone text messaging (txt2stop): a single-blind, randomized trial. Lancet (London, England), 378(9785), 49-55. https://doi.org/10.1016/S0140-6736(11)60701-0

Edwards, A. M., Mann, M. E., Marfell-Jones, M. J., Rankin, D. M., Noakes, T. D., \& Shillington, D. P. (2007). Influence of moderate dehydration on soccer performance: physiological responses to $45 \mathrm{~min}$ of outdoor match-play and the immediate subsequent performance of sport-specific and mental concentration tests. British Journal of Sports Medicine, 41(6), 385-391. https://doi.org/10.1136/bjsm.2006.033860

Evans, G. H., James, L. J., Shirreffs, S. M., \& Maughan, R. J. (2017). Optimizing the restoration and maintenance of fluid balance after exercise-induced dehydration. Journal of Applied Physiology (Bethesda, Md. : 1985), 122(4), 945-951. https://doi.org/10.1152/japplphysiol.00745.2016

Hew-Butler, T., Loi, V., Pani, A., \& Rosner, M. H. (2017). Exercise-Associated Hyponatremia: 2017 Update. Frontiers in Medicine, 4, 21. https://doi.org/10.3389/fmed.2017.00021

Judge, L. W., Kumley, R. F., Bellar, D. M., Pike, K. L., Pierson, E. E., Weidner, T., Pearson, D., \& Friesen, C. A. (2016). Hydration and Fluid Replacement Knowledge, Attitudes, Barriers, and Behaviors of NCAA Division 1 American Football Players. Journal of Strength and Conditioning Research, 30(11), 2972-2978. https://doi.org/10.1519/JSC.0000000000001397

Kenefick R. W. (2018). Drinking Strategies: Planned Drinking Versus Drinking to Thirst. Sports Medicine (Auckland, N.Z.), 48(Suppl 1), 31-37. https://doi.org/10.1007/s40279-0170844-6 
Magee, P. J., Gallagher, A. M., \& McCormack, J. M. (2017). High Prevalence of Dehydration and Inadequate Nutritional Knowledge Among University and Club Level Athletes. International journal of Sport Nutrition and Exercise Metabolism, 27(2), 158-168. https://doi.org/10.1123/ijsnem.2016-0053

McDermott, B. P., Anderson, S. A., Armstrong, L. E., Casa, D. J., Cheuvront, S. N., Cooper, L., Kenney, W. L., O'Connor, F. G., \& Roberts, W. O. (2017). National Athletic Trainers' Association Position Statement: Fluid Replacement for the Physically Active. Journal of Athletic Training, 52(9), 877-895. https://doi.org/10.4085/1062-6050-52.9.02

McDermott, B. P., Casa, D. J., Ganio, M. S., Lopez, R. M., Yeargin, S. W., Armstrong, L. E., \& Maresh, C. M. (2009). Acute whole-body cooling for exercise-induced hyperthermia: a systematic review. Journal of Athletic Training, 44(1), 84-93. https://doi.org/10.4085/1062-6050-44.1.84

Nichols, P. E., Jonnalagadda, S. S., Rosenbloom, C. A., \& Trinkaus, M. (2005). Knowledge, attitudes, and behaviors regarding hydration and fluid replacement of collegiate athletes. International Journal of Sport Nutrition and Exercise Metabolism, 15(5), 515-527. https://doi.org/10.1123/ijsnem.15.5.515

Nuccio, R. P., Barnes, K. A., Carter, J. M., \& Baker, L. B. (2017). Fluid Balance in Team Sport Athletes and the Effect of Hypohydration on Cognitive, Technical, and Physical Performance. Sports Medicine (Auckland, N.Z.), 47(10), 1951-1982. https://doi.org/10.1007/s40279-017-0738-7

Olzinski, S., Beaumont, J., Toledo, M., Yudell, A., Johnston, C. S., \& Wardenaar, F. C. (2019). Hydration Status and Fluid Needs of Division I Female Collegiate Athletes Exercising Indoors and Outdoors. Sports, 7(7), 155. doi:10.3390/sports7070155 
Orrù, S., Imperlini, E., Nigro, E., Alfieri, A., Cevenini, A., Polito, R., Daniele, A., Buono, P., \& Mancini, A. (2018). Role of Functional Beverages on Sport Performance and Recovery. Nutrients, 10(10), 1470. https://doi.org/10.3390/nu10101470

Osterberg, K. L., Horswill, C. A., \& Baker, L. B. (2009). Pregame urine specific gravity and fluid intake by National Basketball Association players during competition. Journal of athletic training, 44(1), 53-57. https://doi.org/10.4085/1062-6050-44.1.53

Savoie, F. A., Kenefick, R. W., Ely, B. R., Cheuvront, S. N., \& Goulet, E. D. (2015). Effect of Hypohydration on Muscle Endurance, Strength, Anaerobic Power and Capacity and Vertical Jumping Ability: A Meta-Analysis. Sports Medicine (Auckland, N.Z.), 45(8), 1207-1227. https://doi.org/10.1007/s40279-015-0349-0

Schwebel, F. J., \& Larimer, M. E. (2018). Using text message reminders in health care services: A narrative literature review. Internet Interventions, 13, 82-104. https://doi.org/10.1016/j.invent.2018.06.002

Sobana, R. M., \& Many, J. N. (2014). Effect of Hydration Education on College Athlete's Hydration Status and KAP. International Journal of Science and Research, 3(7). Trakman, G. L., Brown, F., Forsyth, A., \& Belski, R. (2019). Modifications to the nutrition for sport knowledge questionnaire (NSQK) and abridged nutrition for sport knowledge questionnaire (ANSKQ). Journal of the International Society of Sports Nutrition, 16(1), 26. https://doi.org/10.1186/s12970-019-0293-8

Williams, J., Tzortziou Brown, V., Malliaras, P., Perry, M., \& Kipps, C. (2012). Hydration strategies of runners in the London Marathon. Clinical journal of Sport Medicine: Official Journal of the Canadian Academy of Sport Medicine, 22(2), 152-156. https://doi.org/10.1097/JSM.0b013e3182364c45 
Yoshida, T., Takanishi, T., Nakai, S., Yorimoto, A., \& Morimoto, T. (2002). The critical level of water deficit causing a decrease in human exercise performance: a practical field study. European Journal of Applied Physiology, 87(6), 529-534.https://doi.org/10.1007/s00421002-0651-z 


\section{APPENDIX A: HYDRATION KNOWLEDGE QUESTIONNAIRE}

1. Salt tablets prevent dehydration during competition training $\quad T / F$

2. Thirst is the best indicator of dehydration $\quad T / F$

3. Dehydration decreases athletic performance T/F

4. Athletes should not drink water or fluids during practice $\quad T / F$

5. Coaches should not let players drink fluids during practice $\quad T / F$

6. Coaches should not let players drink fluids during competition T/F

7. It is important for fluids to be readily available to athletes during practice T/F

8. It is important to have fluids readily available to athletes during competition T/F

9. Athletes should drink sports drinks within 2 hours after exercise $\quad T / F$

10. Sports drinks are better than water because they restore muscle glycogen $\quad T / F$

11. An athlete should drink 7-10 fl oz of water or sports drink a couple hours before exercise $\quad T / F$

12. When exercising more than 1 hour an athlete should drink a sports drink over water $\quad T / F$

13. Monitoring urine color is a way to judge if an athlete is hydrated T/F

14. Weighing before and after practice is a good indicator of how much fluid to consume $\quad T / F$

15. Excessive sweating, thirst, \& cramping are signs of dehydration $\quad T / F$

16. More than 2 alcoholic drinks the day before competition can lead to dehydration $\quad T / F$ 


\section{APPENDIX B: HYDRATION BEHAVIOR QUESTIONNAIRE \\ Defining the behavior: Hydration}

Developing adequate hydration practices before, during, and after training for 6 weeks

\section{Specifying the research population:}

The population of interest is the ISU women's soccer team

\section{Attitude:}

1. Drinking a large amount of water 3-4 hours before training would be

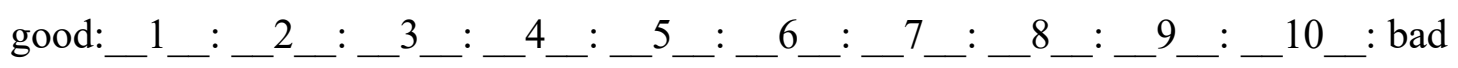

2. Drinking a sports drink during practices that last longer than an hour would be

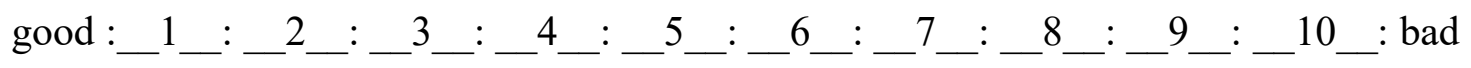

\section{Perceived norm:}

3. My coaches encourage me to drink more water so that I am not dehydrated during practice or games

agree :_1_:_2_:_3_:_4_:_5_:_6_: 7_:_8_:_9_:_10_: disagree 
4. Most of my teammates begin practice in an already hydrated state

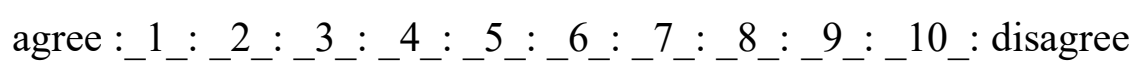

\section{Perceived behavioral control:}

5. I am confident that I consume adequate amounts of water to avoid becoming dehydrated during practice

true :_1_: 2 _ $:{ }_{-}{ }_{-}:{ }_{-}{ }_{-}:{ }_{-}{ }_{-}:{ }_{-}{ }_{-}:{ }_{-}{ }_{-}:{ }_{-}{ }_{-}:{ }_{-}{ }_{-}:{ }_{-} 10 \_$false

6. It is up to me whether or not I consume water before, during, and after training

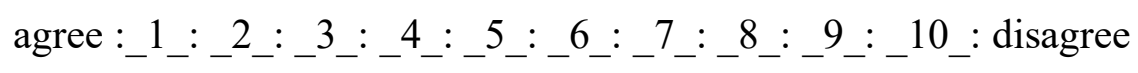

\section{Intention:}

7. I intend to consume fluids before, during, and after training sessions

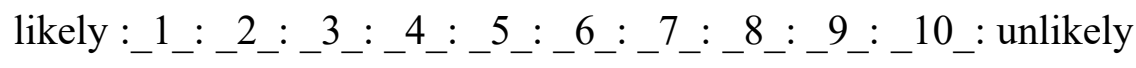

8. I intend to consume some type of electrolyte containing beverage after a training session 
likely :_1_:_2_:_3_:_4_:_5_:_6_:_7_:_8_:_9_:_10_: unlikely

\section{Past behavior:}

9. In the past season I drank enough fluid to avoid being dehydrated during training

true :_1_:_2_: 3_:_4_: 5_:_6_: 7_:_8_:_9_:_10_: false

10. In the past season I have experienced symptoms of dehydration during several training sessions

true :_1_:_2_:_3_:_4_:_5_:_6_:_7_:_8_:_9_:_10_: false 